\title{
Tecnologia de aplicação de agrotóxicos no controle de fitonematoides em soja
}

\author{
Technology application technology of pesticides for control of soybean nematodes
}

\author{
Gerson Dalla Corte $^{I^{*}}$ Felipe Frigo Pinto $^{\mathrm{I}}$ Marlon Tagliapietra Stefanello ${ }^{\mathrm{I}}$ Caroline Gulart ${ }^{\mathrm{I}}$ \\ Juliano Perlin de Ramos ${ }^{\mathrm{I}}$ Ricardo Silveiro Balardin ${ }^{\mathrm{I}}$
}

\section{RESUMO}

O objetivo deste trabalho foi avaliar o efeito de diferentes sistemas de aplicação tratamento de sementes (TS), sulco de semeadura (Sulco) e associação de ambos (TS + Sulco) e tratamentos químicos no controle de Pratylenchus brachyurus e Meloydogine javanica, em condição de campo e ambiente controlado em vaso. O delineamento utilizado foi de blocos ao acaso, com parcelas subdivididas, em que, nas parcelas principais, foram alocados três sistemas de aplicação: tratamento de sementes, aplicação no sulco + tratamento de sementes (duas vezes a dose) e aplicação no sulco. As subparcelas foram constituídas de diferentes tratamentos químicos: testemunha; abamectina + tiametoxam + fludioxonil + metalaxil- $M+$ tiabendazol $\left(30+42+1,5+1,2+9 g\right.$ i.a. $\left.h^{-1}\right)$; imidacloprido + tiodicarbe + carbendazim + tiram $\left(63+189+18+42 \mathrm{~g}\right.$ i.a. ha $\left.\mathrm{h}^{-1}\right) ;$ fipronil + tiofanato-metílico + piraclostrobina $\left(30+27+3 g\right.$ i.a. $\left.h^{-1}\right)$; carbofurano + carbendazim + tiram $\left(420+18+42\right.$ g i.a. ha $\left.a^{-1}\right)$. Avaliou-se a população de fitonematoides no sistema radicular (campo e ambiente controlado), área foliar e massa seca de parte aérea (ambiente controlado). A associação de TS + sulco resultou em maior eficácia e efeito residual de controle dos nematoides. O tratamento com abamectina + tiametoxam + fludioxonil + metalaxil-M + tiabendazol promoveu os maiores níveis de controle, com benefícios expressivos no desenvolvimento das plantas.

Palavras-chave: Pratylenchus brachyurus, Meloydogyne javanica, controle químico, tratamento de semente, sulco.

\section{ABSTRACT}

This study aimed to evaluate the effect of different application systems seed treatment (ST), in-furrow application and association of both (ST + in-furrow) and chemical treatments for control of Pratylenchus brachyurus and Meloydogine javanica under field condition and controlled atmosphere in pots. The experimental design was a randomized block, with split plot and four replications. In the main plots were allocated three application systems: seed treatment, in-furrow + seed treatment (double dose) and in-furrow. The subplots were composed of different chemical treatments: check, abamectin + thiamethoxam + fludioxonil + metalaxyl- $M+$ thiabendazole $(30+42+9+1.5+1.2 \mathrm{~g}$ a.i. $\left.\mathrm{ha}^{-1}\right)$, imidacloprid + thiodicarb + carbendazim + thiram $\left(63+189+18+42 g\right.$ a.i. $\left.\mathrm{ha}^{-1}\right)$, fipronil + thiophanate-methyl + pyraclostrobin $\left(30+27+3 g\right.$ a.i. ha $\left.^{-1}\right)$, carbofuran + carbendazim + thiram $\left(420+18+42 \mathrm{~g}\right.$ a.i. $\left.\mathrm{ha}^{-1}\right)$. It was evaluated the population of nematodes in the roots (field and controlled atmosphere), leaf area and shoot dry weight (controlled atmosphere). It was found that association of TS + in-furrow application provided higher efficacy and residual effect for nematode control under field and controlled atmosphere conditions. The treatment with abamectin + thiamethoxam + fludioxonil + metalaxyl-M + thiabendazole delivered greatest levels of control, with significant benefits in plant development.

Key words: Pratylenchus brachyurus, Meloydogyne javanica, chemical control, seed treatment, in-furrow.

\section{INTRODUÇÃO}

Os problemas fitossanitários estão entre os principais fatores que limitam a obtenção de altos rendimentos em soja, destacando-se os nematoides como patógenos responsáveis por prejuízos crescentes (ALMEIDA et al., 2005). Dentre os gêneros de maior importância e interesse econômico para a soja, destacam-se as espécies Meloidogyne javanica e Meloidogyne incognita (nematoides de galhas) e Pratylenchus brachyurus (nematoide das lesões radiculares) (FERRAZ, 2001). O ataque desses nematoides resulta muitas vezes na redução do tamanho das plantas, com intenso abortamento de vagens durante o florescimento e amadurecimento prematuro das plantas,

\footnotetext{
${ }^{\text {I}}$ Departamento de Defesa Fitossanitária, Universidade Federal de Santa Maria (UFSM), CP 5025, 97111-970, Santa Maria, RS, Brasil. E-mail: gerson.agro@gmail.com.*Autor para correspondência. 
podendo os danos variarem de 30 a $50 \%$ em função da intensidade do ataque (GOULART, 2008; DIAS, 2010).

A erradicação de nematoides em área infestada é extremamente difícil. Entre as estratégias de manejo integrado consideradas mais promissoras, podem ser citadas a rotação ou sucessão de culturas com espécies não hospedeiras, a utilização de cultivares resistentes ou tolerantes e o tratamento químico (ARAÚJO et al., 2012). O manejo químico de nematoides pode ser feito com o uso de nematicidas no tratamento de sementes (CABRERA et al., 2009) ou em aplicação no sulco de semeadura (NOVARETTI; REIS, 2009), embora na cultura da soja esta técnica ainda seja muito pouco difundida.

No entanto, os resultados de controle de nematoides via tratamento de semente apontam curto período de proteção, chegando até 30 dias após a emergência das plantas, na grande maioria dos casos (FASKE \& STARR, 2007; CABRERA et al., 2009). Esse fato pode estar atrelado às restrições impostas pela tecnologia de aplicação. Uma possibilidade seria a aplicação de defensivos líquidos no sulco de semeadura ou a associação de tratamento de sulco e tratamento de sementes, o que em partes poderia minimizar as deficiências inerentes ao tratamento de sementes. Entretanto, esta técnica praticamente ainda não foi estudada no Brasil para a cultura da soja, consequentemente há uma grande escassez de trabalhos mostrando resultados que comprovem a sua eficácia.

Dessa forma, o objetivo deste trabalho foi avaliar o efeito de diferentes sistemas de aplicação de agroquímicos tratamento de sementes (TS), sulco de semeadura (Sulco) e associação dos dois (TS + Sulco) e tratamentos químicos (inseticidas/nematicidas) no controle de Pratylenchus brachyurus e Meloydogine javanica e desenvolvimento da cultura da soja em condição de campo e ambiente controlado.

\section{MATERIAL E MÉTODOS}

O experimento de campo foi instalado em uma unidade de produção comercial de soja, localizada no município de Júlio de Castilho - RS, com consistente histórico de perdas decorrentes da ocorrência de fitonematoides na cultura da soja, nos últimos cinco anos. O delineamento experimental utilizado foi de blocos ao acaso em parcelas subdivididas, em arranjo fatorial $(3 \times 5)$ e quatro repetições. Nas parcelas principais, foram alocados os sistemas de aplicação (tratamento de semente, líquido no sulco e a associação destes) e nas subparcelas os tratamentos químicos acrescido de uma testemunha, sem aplicação de produto (Tabela 1).

Tabela 1 - Sistemas de aplicação, tratamentos químicos (produto comercial), princípios ativos e doses dos produtos avaliados para o controle de fitonematoides da soja em nível de campo. Santa Maria, UFSM, 2012.

\begin{tabular}{|c|c|c|c|}
\hline $\begin{array}{l}\text { Sistemas de } \\
\text { aplicação }\end{array}$ & Tratamentos químicos & Princípios ativos & Doses (g de i.a. ha $^{-1}$ ) \\
\hline \multirow{5}{*}{$\mathrm{TS}^{(1)}$} & Testemunha & - & - \\
\hline & Avicta Completo & $\begin{array}{l}\text { abamectina }+ \text { tiametoxam }+ \text { fludioxonil }+ \text { metalaxil-M }+ \\
\text { tiabendazol }\end{array}$ & $30+42+1,5+1,2+9$ \\
\hline & Cropstar + Derosal Plus & imidacloprido + tiodicarbe + carbendazim + tiram & $63+189+18+42$ \\
\hline & Standak Top & fipronil + tiofanato-metílico + piraclostrobina & $30+27+3$ \\
\hline & $\begin{array}{l}\text { Furadan } 350 \text { FS + Derosal } \\
\text { Plus }\end{array}$ & carbofurano + carbendazim + tiram & $420+18+42$ \\
\hline \multirow{5}{*}{ TS + Sulco ${ }^{(2)}$} & Testemunha & - & - \\
\hline & Avicta Completo & $\begin{array}{l}\text { abamectina }+ \text { tiametoxam }+ \text { fludioxonil }+ \text { metalaxil-M }+ \\
\text { tiabendazol }\end{array}$ & $30+42+1,5+1,2+9$ \\
\hline & Cropstar + Derosal Plus & imidacloprido + tiodicarbe + carbendazim + tiram & $63+189+18+42$ \\
\hline & Standak Top & fipronil + tiofanato-metílico + piraclostrobina & $30+27+3$ \\
\hline & $\begin{array}{l}\text { Furadan } 350 \text { FS + Derosal } \\
\text { Plus }\end{array}$ & carbofurano + carbendazim + tiram & $420+18+42$ \\
\hline \multirow{5}{*}{ Sulco ${ }^{(3)}$} & Testemunha & - & - \\
\hline & Avicta Completo & $\begin{array}{l}\text { abamectina }+ \text { tiametoxam }+ \text { fludioxonil }+ \text { metalaxil-M }+ \\
\text { tiabendazol }\end{array}$ & $30+42+1,5+1,2+9$ \\
\hline & Cropstar + Derosal Plus & imidacloprido + tiodicarbe + carbendazim + tiram & $63+189+18+42$ \\
\hline & Standak Top & fipronil + tiofanato-metílico + piraclostrobina & $30+27+3$ \\
\hline & $\begin{array}{l}\text { Furadan } 350 \text { FS + Derosal } \\
\text { Plus }\end{array}$ & carbofurano + carbendazim + tiram & $420+18+42$ \\
\hline
\end{tabular}

\footnotetext{
${ }^{(1)}$ Tratamento de sementes. ${ }^{(2)}$ Tratamento de sementes associado à aplicação de inseticidas/nematicidas pulverizado em jato dirigido no sulco de semeadura. ${ }^{(3)}$ Aplicação de inseticidas/nematicidas em jato dirigido no sulco de semeadura.
} 
A semeadura foi realizada com o auxílio de uma semeadora Imasa PHS 63, dotada de três linhas, espaçadas a $50 \mathrm{~cm}$, sendo esta tracionada por um trator Landini, modelo Montana 45, a uma velocidade de $5,4 \mathrm{~km} \mathrm{~h}^{-1}$. Para a aplicação dos tratamentos no sulco, foi adaptado na semeadora um equipamento pressurizado a $\mathrm{CO}_{2}$, dotado de um sistema de mangueiras e pontas do tipo cone vazio TX 80 015, mais adequada para esse tipo de aplicação em função do seu padrão de distribuição da calda e menor de entupimento por partículas de solo. As mangeiras foram acopladas junto às linhas de semeadura, atrás da haste sulcadora de fertilizantes, o que permitiu a aplicação direta dos produtos no interior do sulco no solo. O conjunto de pontas foi instalado em uma posição que, quando em trabalho, as mesmas ficavam praticamente na altura da linha do solo, fazendo com que a totalidade do produto fosse depositada no interior do sulco. A faixa de deposição foi de aproximadamente $0,07 \mathrm{~m}$ de largura e profundidade, chegando a aproximadamente $0,15 \mathrm{~m}$. O equipamento foi calibrado a uma pressão de trabalho de $300 \mathrm{KPa}$ e volume de calda de $80 \mathrm{~L} \mathrm{ha}^{-1}$.

A cultivar utilizada foi a 'Syngenta Vmax RR', com ciclo de maturação 6.2, suscetível aos nematoides causadores de galhas e de lesões radiculares. A semeadura foi efetuada no dia 12 de novembro de 2011, utilizando-se um espaçamento de $0,50 \mathrm{~m}$ entre fileiras, profundidade de $0,03 \mathrm{~m}$ e uma densidade de 28 plantas $\mathrm{m}^{-2}$, o que representou um total de $60 \mathrm{~kg}$ de semente por hectare. A adubação de base utilizada foi de $400 \mathrm{~kg} \mathrm{ha}^{-1}$ da fórmula NPK 5-25-25, de acordo com a análise do solo, e os demais tratos culturais foram realizadas conforme as recomendações para a cultura da soja (REUNIÃO..., 2010).

$\mathrm{O}$ tratamento de sementes foi realizado em sacos plásticos com capacidade de $3 \mathrm{~L}$, utilizandose uma amostra de $1 \mathrm{~kg}$ de semente por tratamento. Os produtos foram dosados com seringas graduadas de $3 \mathrm{~mL}$, sendo o volume completado com água até um valor compatível a $800 \mathrm{~mL}$ de calda por $100 \mathrm{~kg}$ de sementes, para garantir a uniformidade de distribuição dos produtos na superfície das sementes.

$\mathrm{O}$ experimento em ambiente controlado foi conduzido em casa-de-vegetação da estação experimental do Instituto Phytus, município de Itaara - RS, dotada de um sistema de controle de temperatura, umidade e fertirrigação automatizados. Para a semeadura, foram utilizados vasos plásticos de $5 \mathrm{~L}$, preenchidos com substrato a base de areia e solo, previamente esterilizados, na proporção 1:1, com a cultivar de soja 'Syngenta Vmax RR'. O delineamento experimental de blocos ao acaso, em esquema bifatorial $(3 \times 5)$ e cinco repetições, cujos fatores e os tratamentos foram os mesmos utilizados no experimento de campo (Tabela 1). Cada unidade experimental foi constituída por um vaso, com apenas uma planta.

Para o tratamento de sementes, foi utilizado o mesmo procedimento descrito acima, no experimento conduzido em nível de campo. A aplicação dos tratamentos no sulco foi realizada no momento da semeadura, utilizando-se um pulverizador costal pressurizado a $\mathrm{CO}_{2}$, dotado de lança simples com ponta de pulverização do tipo TX 80015 e calibrado à pressão de $300 \mathrm{KPa}$ e vazão de trabalho de $80 \mathrm{~L} \mathrm{ha}^{-1}$. O sulco foi aberto manualmente a $0,15 \mathrm{~m}$ de profundidade e $0,07 \mathrm{~m}$ de largura, a fim de possibilitar a deposição dos tratamentos no interior do solo. $\mathrm{O}$ jato de aplicação cobriu completamente a área interna do sulco de semeadura, que, após a aplicação, foi remanejado para $0,03 \mathrm{~m}$ de profundidade para então efetuar-se a deposição das sementes. Aos cinco dias após a emergência (DAE), cada planta foi inoculada com $4 \mathrm{~mL}$ de uma suspensão de 1.000 indivíduos (ovos + juvenis) $\mathrm{mL}^{-1}$ de uma população de Meloidogyne javanica proveniente de Júlio de Castilho - RS, extraído de vários sistemas radiculares de plantas de soja da cultivar 'M-soy 8000 RR', com base na técnica descrita por BONETI \& FERRAZ (1981). A suspensão de ovos + juvenis foi vertida em dois orifícios de $2 \mathrm{~cm}$ de profundidade e distantes $2 \mathrm{~cm}$ do colo da planta.

Os parâmetros avaliados foram níveis populacionais de fitonematoides no sistema radicular das plantas aos 30 e 90DAE no campo e aos 30 e 60DAE nos vasos, seguindo a metodologia descrita por BONETI \& FERRAZ (1981). Além disso, foram avaliadas a massa seca de parte aérea e área foliar aos 30 e 60DAE nos vasos. Para estimativa da área foliar, utilizou-se o software QUANT ${ }^{\circledR}$ (VALE et al., 2001).

Para efeito de análise estatística, os dados de nematoides obtidos no sistema radicular foram transformados em $\sqrt{X+0,5}$ devido às médias e os erros destes não seguirem distribuição normal, e posteriormente junto com os demais foram submetidos à análise de variância (teste $\mathrm{F} \leq 5 \%$ ) e as médias também foram comparadas pelo teste de Tukey (5\%), calculado através do software ASSITAT, versão 7.6 (SILVA \& AZEVEDO, 2009).

\section{RESULTADOS E DISCUSSÃO}

O levantamento prévio da população de nematoides, realizado na área do experimento de campo, detectou uma infestação média de 202 indivíduos (J2) de Meloidogyne javanica, 279 indivíduos (J2) de Helicotilenchus sp., 3,31

Ciência Rural, v.44, n.9, set, 2014. 
indivíduos (J2) de Pratylenchus brachyurus e 3,44 ovos a cada $250 \mathrm{~cm}^{3}$ de solo.

A análise de variância mostrou interação significativa entre os sistemas de aplicação e tratamentos químicos para os níveis populacionais de Pratylenchus brachyurus e Meloidogyne javanica aos 30DAE e 90DAE em campo e para Meloidogyne javanica aos 30 e 60DAE em vasos, ao nível de 5\% de significância (Tabela 2 e 3). De maneira geral, considerando-se apenas os sistemas de aplicação, não houve diferença estatística em nenhuma das avaliações de campo e vasos, mostrando que a resposta da tecnologia de aplicação está condicionada às características específicas de cada produto.
Quando comparados os desempenhos de controle dos tratamentos químicos para Pratylenchus brachyurus em nível de campo aos 30DAE, observase que eles apresentaram diferenças significativas da testemunha somente quando aplicados via TS + Sulco ou Sulco. Nas duas formas de aplicação, o maior nível de controle foi verificado no tratamento abamectina + tiametoxam + fludioxonil + metalaxil-M + tiabendazol, diferindo estatisticamente da testemunha. Amagnitude do controle desse tratamento foi de 70,9\% e 64,5\%, quando aplicados via TS + Sulco e Sulco, respectivamente. Do mesmo modo, o tratamento abamectina + tiametoxam + fludioxonil + metalaxil-M + tiabendazol destacou-se frente aos demais nos três sistemas de aplicação para

Tabela 2 - Níveis populacionais de Pratylenchus brachyurus e Meloidogyne javanica em 5g de raízes de soja conduzidas em condições de campo aos 30 e 90DAE, em função de diferentes tratamentos químicos e sistemas de aplicação.

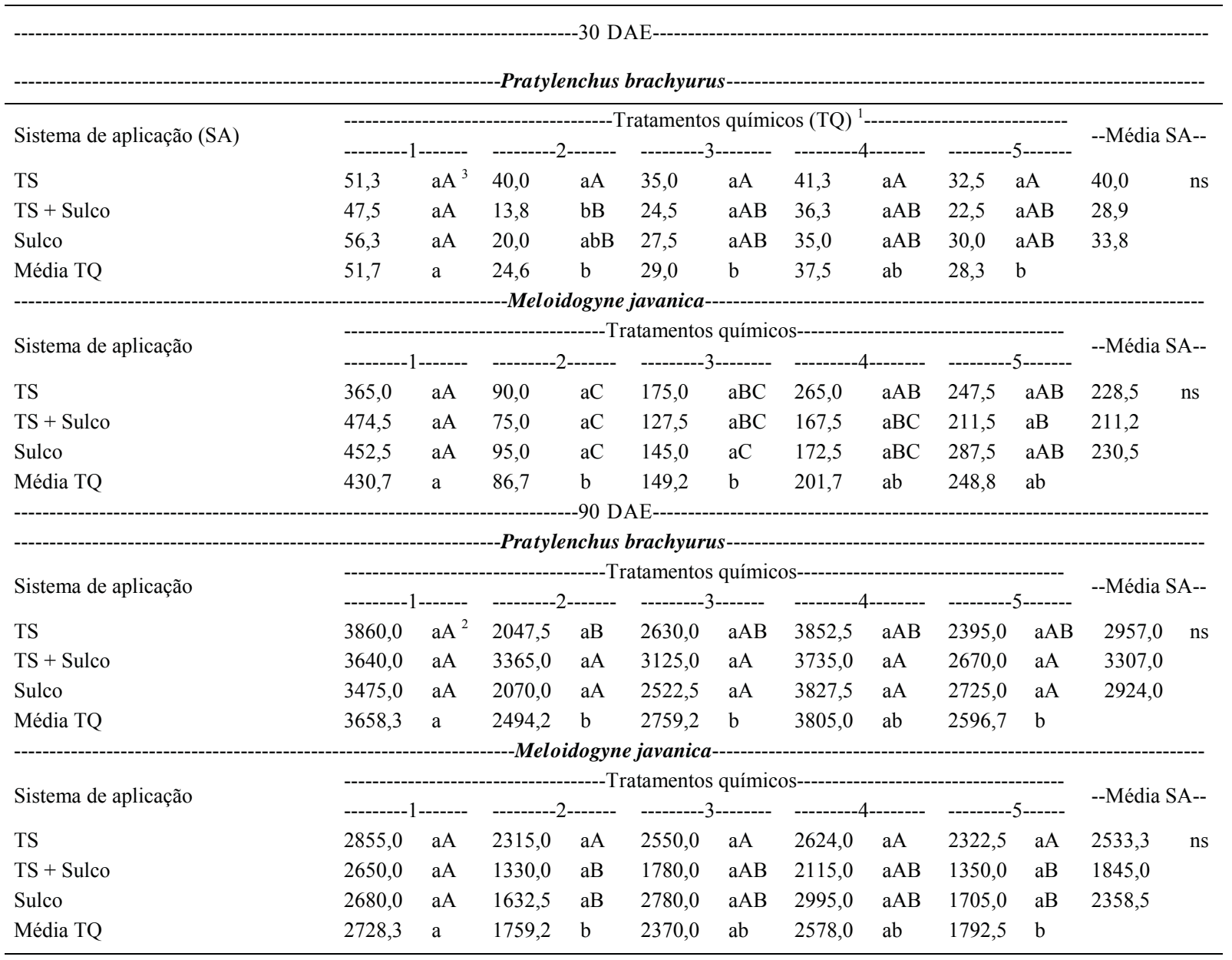

${ }^{1}$ Tratamentos químicos: 1 - Testemunha; 2 - Avicta Completo (abamectina + tiametoxam + fludioxonil + metalaxil-M + tiabendazol $-30+$ $42+1,5+1,2+9$ g i.a. ha $^{-1}$ ) $; 3-$ Cropstar + Derosal Plus (imidacloprido + tiodicarbe + carbendazim + tiram $-63+189+18+42 \mathrm{~g}$ i.a. ha ${ }^{-1}$ ) 4 - Standak Top (fipronil + tiofanato-metílico + piraclostrobina $-30+27+3 \mathrm{~g}$ i.a. ha ${ }^{-1}$ ); 5 - Furadan 350 FS + Derosal Plus (carbofurano + carbendazim + tiram $-420+18+42 \mathrm{~g}$ i.a. ha $\left.{ }^{-1}\right) .{ }^{2}$ Médias seguidas pela mesma letra maiúscula nas linhas e minúscula nas colunas não diferem estatisticamente entre si pelo teste de Tukey em nível de 5\% de probabilidade de erro. Coeficientes de variação (\%) 30DAE: Pratylenchus brachyurus $=38,82$ e Meloidogyne javanica $=47,21$. Coeficientes de variação $(\%)$ 90DAE: Pratylenchus brachyurus $=$ 31,56; Meloidogyne javanica $=65,21$. 
Tabela 3 - Nível populacional de Meloidogyne javanica em $5 \mathrm{~g}$ de raízes de soja conduzidas em vasos aos 30 e 60DAE, em função de diferentes tratamentos químicos e sistemas de aplicação.

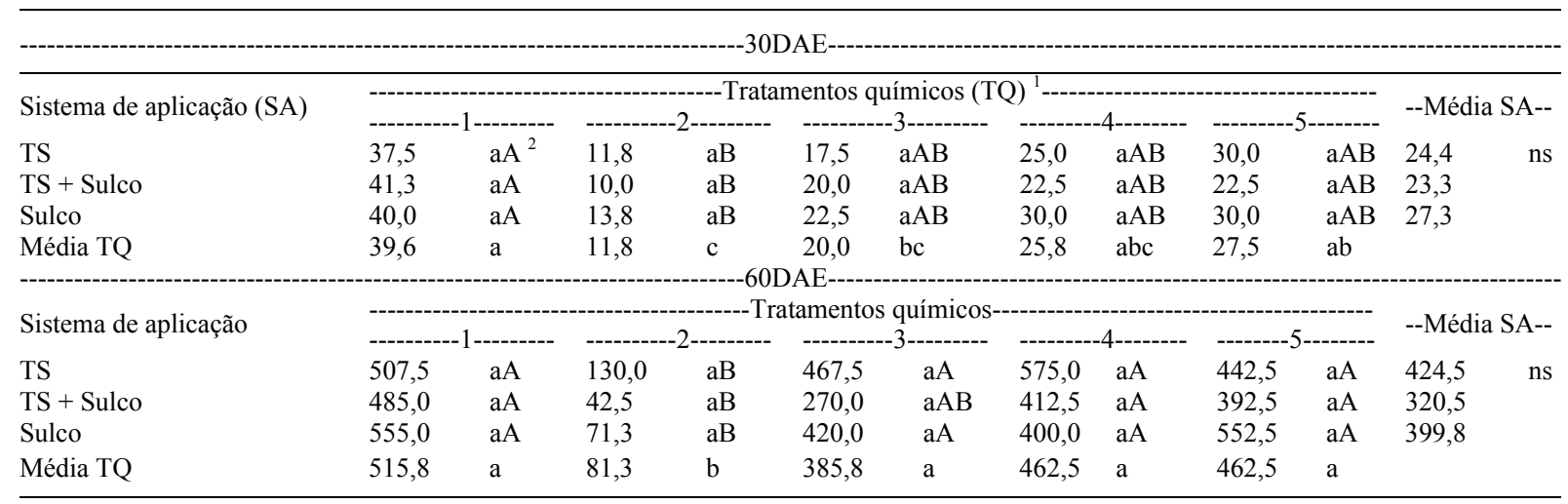

${ }^{1}$ Tratamentos químicos: 1 - Testemunha; 2 - Avicta Completo (abamectina + tiametoxam + fludioxonil + metalaxil-M + tiabendazol $-30+$

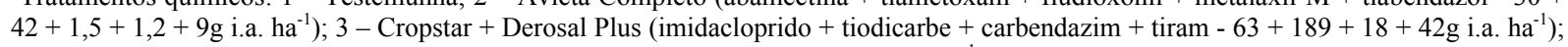
4 - Standak Top (fipronil + tiofanato-metílico + piraclostrobina $-30+27+3 \mathrm{~g}$ i.a. ha ${ }^{-1}$ ); 5 - Furadan 350 FS + Derosal Plus (carbofurano + carbendazim + tiram $-420+18+42 \mathrm{~g}$ i.a. ha $\left.{ }^{-1}\right) .{ }^{2}$ Médias seguidas pela mesma letra maiúscula nas linhas e minúscula nas colunas não diferem estatisticamente entre si pelo teste de Tukey em nível de 5\% de probabilidade de erro. Coeficientes de variação $(\%): 30 \mathrm{DAE}=42,45$ e $60 \mathrm{DAE}=42,86$.

Meloidogyne javanica aos 30DAE, com controles na ordem de $75,4 \%, 84,2 \%$ e 78,9\%, quando aplicados via TS, TS + Sulco e Sulco, respectivamente. (Tabela 2).

A pequena diferença de controle entre os sistemas de aplicação para os dois nematoides aos 30 DAE pode ter uma explicação em que a quantidade de ativo aplicada e o local de deposição do ativo tanto via TS como Sulco pode ter sido suficiente para garantir um desempenho semelhante à associação de TS + Sulco. Na avaliação de 30DAE, verificaram-se níveis mais elevados de controle de Meloidogyne javanica em relação a Pratylenchus brachyurus, seguindo a mesma tendência observada por VITTI (2009), que obteve efetiva redução populacional de Heterodera glycines com o tratamento de sementes a base de abamectina, sem apresentar efeito significativo sobre a redução da população de Pratylenchus brachyurus, indicando a maior dificuldade de controle desses nematoides quando comparados aos demais que incidem na cultura da soja. Essa especificidade pode ter, como uma das possíveis explicações, além das diferenças de sensibilidade aos produtos químicos intrínsecas de cada espécie de nematoide, o fato do Pratylenchus brachyurus ser um nematoide de hábito migrador e assim não formar um sítio de alimentação permanente com a raiz, como é característico dos nematoides dos gêneros Meloidogyne spp. e Heterodera spp..

Já os níveis de controle observados aos 90DAE foram inferiores aos observados nos 30DAE, demonstrando perda de desempenho ou efeito residual dos tratamentos químicos com o avanço do ciclo da cultura em condições de campo (Tabela 2). A magnitude da queda de controle foi menos acentuada nos tratamentos cuja aplicação foi realizada na forma de TS + Sulco, indicando o benefício da maior deposição e melhor distribuição do ativo dos produtos no perfil do sulco de semeadura. Considerando as aplicações isoladas de TS e Sulco, pode-se observar maior controle da aplicação via Sulco aos 90DAE. Esse fato reforça ainda mais a importância da tecnologia de aplicação quando se fala em controle químico de nematoide, sendo a magnitude dessa resposta dependente muitas vezes da característica do produto (mobilidade no solo e persistência) e do nematoide alvo.

Ao analisar-se os tratamentos químicos para o ensaio conduzido em vasos, pode-se observar que somente o tratamento abamectina + tiametoxam + fludioxonil + metalaxil-M + tiabendazol apresentou diferença significativa em relação à testemunha, independentemente da forma de aplicação (TS, TS + Sulco ou Sulco) aos 30 e 60DAE (Tabela 3). Esse comportamento foi diferenciado do observado no campo, onde a associação TS + Sulco se destacou frente às demais formas de aplicação, em avaliações mais tardias. Uma possível explicação pode estar no fato de que, nas condições de campo, a degradação de ativo é mais intensa quando comparada a um ambiente controlado por ações bióticas e abióticas, e por isso os tratamentos com maior quantidade de ativo (TS + Sulco) promoveram um controle mais prolongado em comparação aos TS e Sulco isolados.

Percebe-se claramente através da análise dos dados de área foliar e massa seca, que o desenvolvimento das plantas está diretamente ligado ao nível de interferência do ataque de Meloidogyne 
javanica, uma vez que os maiores níveis de controle do nematoide refletiram-se em maior desenvolvimento das plantas, medidos indiretamente por essas variáveis (Tabela 4). Com exceção do tratamento a base de fipronil + tiofanato-metílico + piraclostrobina, que não promeveu controle significativo de Meloidogyne javanica e apresentou os maiores incrementos de área foliar e massa seca, o tratamento abamectina + tiametoxam + fludioxonil + metalaxil-M + tiabendazol, novamente destacou-se entre os demais. A magnitude da resposta mais uma vez esteve diretamente associada à tecnologia de aplicação empregada, principalmente no tratamento abamectina + tiametoxam + fludioxonil + metalaxil-M + tiabendazol, que, quando aplicado via TS + Sulco, aumentou o acúmulo de massa seca na ordem de
$21,82 \%$ e $18,2 \%$ na área foliar, quando comparados à testemunha sem controle.

Em contraste com os resultados encontrados neste trabalho, CABRERA et al. (2009) não observaram diferenças significativas no peso de raízes e parte aérea entre plantas tratadas com abamectina e plantas sem tratamento químico de Pratylenchus zeae em milho e Meloidogyne incognita em algodão. No entanto, deve-se fazer uma ressalva que a tecnologia de aplicação via tratamento de semente, pode ter sido um fator limitante à obtenção de resultados mais satisfatórios. Os autores FASKE \& STARR (2007) verificaram que a grande maioria da abamectina aplicada via tratamento de sementes permanece aderida ao tegumento das sementes. Quando as raízes crescem para fora dessa

Tabela 4 - Área foliar $\left(\mathrm{cm}^{2}\right.$ planta $\left.^{-1}\right)$ e massa seca da parte aérea (g.planta $\left.{ }^{-1}\right)$ de plantas de soja conduzidas em vasos 30 e 60 dias após a emergência (DAE), em função de diferentes tratamentos químicos e sistemas de aplicação.

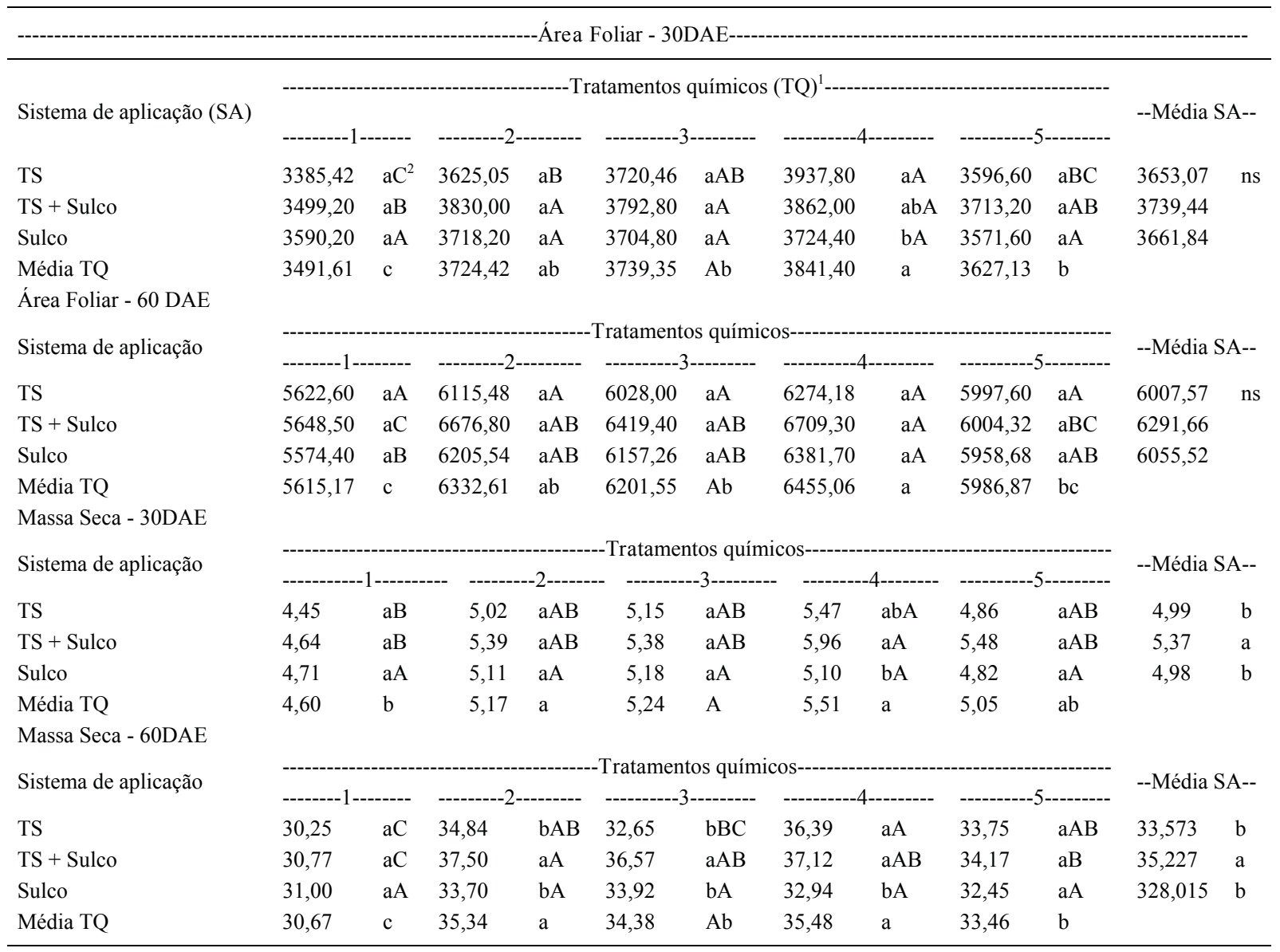

${ }^{1}$ Tratamentos químicos: 1 - Testemunha; 2 - Avicta Completo (abamectina + tiametoxam + fludioxonil + metalaxil-M + tiabendazol $-30+$ $42+1,5+1,2+9 \mathrm{~g}$ i.a. ha $\left.^{-1}\right) ; 3-$ Cropstar + Derosal Plus (imidacloprido + tiodicarbe + carbendazim + tiram $-63+189+18+42 \mathrm{~g}$ i.a. ha ${ }^{-1}$ ) 4 - Standak Top (fipronil + tiofanato-metílico + piraclostrobina - $30+27+3 \mathrm{~g}$ i.a. ha ${ }^{-1}$ ); 5 - Furadan 350 FS + Derosal Plus (carbofurano + carbendazim + tiram $-420+18+42 \mathrm{~g}$ i.a. ha $\left.^{-1}\right)$. ${ }^{2}$ Médias seguidas pela mesma letra maiúscula nas linhas e minúscula nas colunas não diferem estatisticamente entre si pelo teste de Tukey em nível de 5\% de probabilidade de erro. Coeficientes de variação (\%) de área foliar: $30 \mathrm{DAE}=4,49 ; 60 \mathrm{DAE}=7,57$. Coeficientes de variação $(\%)$ de massa seca: $15 \mathrm{DAE}=12,13 ; 30 \mathrm{DAE}=7,11 ; 60 \mathrm{DAE}=3,37$. 
região efetivamente protegida pela abamectina, elas se tornam sensíveis à infecção e colonização de nematoides presentes nesse local. Assim, segundo os autores, uma alternativa para superar essa limitação seria a adoção de uma tecnologia de aplicação que compensasse a imobilidade da abamectina no interior do solo, além da combinação com outros nematicidas sistêmicos e/ou com maior mobilidade no solo.

Com base nos resultados encontrados neste estudo, fica evidente a necessidade de avaliação de novos produtos e modalidades de aplicação para o controle de fitonematoides em soja. Para que isso seja viável, os produtos a serem utilizados, além de seguros em termos toxicológicos e ambientais, devem ser eficientes na redução populacional, além de mitigar seus efeitos deletérios ao desenvolvimento da cultura.

\section{CONCLUSÃO}

A mesma tendência dos resultados encontrados em condições de campo e ambiente controlado nos garante maior consistência em concluir que a associação de TS + Sulco resultou em maior efeito residual de controle, especialmente aos tratamentos abamectina + tiametoxam + fludioxonil + metalaxil-M + tiabendazol e imidacloprido + tiodicarbe + carbendazim + tiram. A tecnologia de aplicação via sulco foi mais efetiva quando comparada ao TS para o tratamento abamectina + tiametoxam + fludioxonil + metalaxil-M + tiabendazol no controle de Meloidogyne javanica. O tratamento com abamectina + tiametoxam + fludioxonil + metalaxil-M + tiabendazol promoveu maiores níveis de controle, com benefícios expressivos no desenvolvimento das plantas. A resposta da tecnologia de aplicação depende das características dos produtos e do nematoide analisado.

\section{REFERÊNCIAS}

ALMEIDA, A.M.R. et al. Doenças da soja. In: KIMATI, H. et al. Manual de fitopatologia. São Paulo: Agronômica Ceres, 2005. p.569-588.

ARAÚJO, F.F. et al. Controle genético, químico e biológico de meloidoginose na cultura da soja. Pesquisa Agropecuária Tropical, v.42, p.220-224, 2012. Disponível em: <http://www. scielo.br/pdf/pat/v42n2/13.pdf>. Acesso em: 25 mai. 2013.
BONETI, J.I.S.; FERRAZ, S. Modificação do método de Hussey e Barker para extração de ovos de Meloidogyne exigua de raízes de cafeeiro. Fitopatologia Brasileira, v.6, p.553, 1981.

CABRERA, J.A. et al. Efficacy of abamectin seed treatment on Pratylenchus zeae, Meloidogyne incognita and Heterodera schachtii. Journal of Plant Diseases and Protection, v.116, 124-128, 2009. Disponível em: <http:// www.cabdirect.org/abstracts/20093193376.html;jsessionid= AA2E073DD5462C65572BAE4249CDE0C0>. Acesso em: 23 mai. 2013.

DIAS, W.P. et al. Nematoides em soja: identificação e controle. Londrina: EMBRAPA, 2010. 8p. (Circular Técnica Embrapa).

FASKE, T.R.; STARR, J.L. Cotton root protection from plantparasitic nematodes by abamectin treated seed. Journal of Nematology, v.39, p.27-30, 2007. Disponível em: <http://www. ncbi.nlm.nih.gov/pmc/articles/PMC2586483/>. Acesso em: 22 mai 2013.

FERRAZ, L.C.C.B. As meloidogynoses da soja: passado, presente e futuro. In: FERRAZ, L.C.C.B. et al. Relações parasito-hospedeiro nas meloidogynoses da soja. Londrina: EMBRAPA/ Sociedade Brasileira de Nematologia, 2001. p.15-38.

GOULART, A.M.C. et al. Aspectos gerais sobre nematoidesdas-lesões radiculares (gênero Pratylenchus). Planaltina: EMBRAPA, 2008. 30p. (Documentos, 219).

NOVARETTI, W.R.T.; REIS, A.M. Influência do método de aplicação de nematicidas no controle de Pratylenchus zeae em soqueiras de cana-de-açúcar e definição dos níveis de dano e de controle. Nematologia Brasileira, v.33, p.8389, 2009. Disponível em: <http://docentes.esalq.usp.br/sbn/ nbonline/ol\%20331/83-89\%20co.pdf>. Acesso em: 22 de abr. 2013.

REUNIÃO DE PESQUISA DE SOJA DA REGIÃO SUL. Indicações técnicas para a cultura da soja no Rio Grande do Sul e em Santa Catarina 2010/2011 e 2011/2012. Cruz Alta, RS: FUNDACEP FECOTRIGO, 2010. 168p.

SILVA, F. de A.S.E.; AZEVEDO, C.A.V. de. Principal components analysis in the software assistat-statistical attendance. In: WORLD CONGRESS ON COMPUTERS IN AGRICULTURA, 7., 2009, Reno. Anais... Reno: American Society of Agricultural and Biological Engineers, 2009.

VALE, F.X.R. et al. Quantificação de doenças - Quant: versão 1.0.1. Viçosa: UFV, 2001. Software.

VITTI, A.J. Tratamento de sementes de soja (Glycine max (L.) Merr.) com abamectina, tiabendazol e acibenzolar-Smetil no manejo de nematoides. 2009, 120f. Tese (Doutorado em Agronomia: Produção Vegetal) - Escola de Agronomia e Engenharia de Alimentos, Universidade Federal de Goiás, Goiânia, Go. 